\title{
Review of: "Emergency responders' experiences of multi-agency working during the COVID-19 response in the UK: Social identity as part of the problem and part of the solution"
}

\author{
Sara Kreindler ${ }^{1}$ \\ 1 University of Manitoba
}

Potential competing interests: I worked with one of the co-authors (Alex Haslam) on some research in the late 2000s/early 2010s.

Thank you for the invitation to review this paper. I wish I didn't have to give a star rating to go with my review - it feels like I've just bought a toaster on Amazon. Since it's necessary, I've sought to express that this is already a good paper, but can be further strengthened prior to publication.

The paper reports an important and timely investigation in a field ripe for application of the social identity approach. I am personally convinced of the importance of social identity to most social/organizational phenomena; however, as part of my due diligence as a reviewer, I will take a critical perspective.

The main area in which I'd like to see the paper strengthened is in its demonstration that social identity processes are at work. Not all social phenomena are social identity phenomena; some are merely interpersonal, some merely reflect practical/operational issues (for lack of a better term). There seems to be a fair amount of prior work (at least external reviews) in this area describing generic issues like communication, coordination, and the need for good leadership and robust information-sharing processes. To make a novel contribution, it is not enough to rebrand these as social identity issues; it's necessary to demonstrate that (1) social categorization and/or group identification are indeed occurring, and not merely some interpersonal or non-social processes, and (2) invoking SIA concepts advances our understanding beyond what we would gain from an atheoretical, common-sense account of the observed phenomena. The study offers some tantalizing hints that SIA processes are at work - mostly in the reported use of collective language by some participants - but many of the themes could be interpreted as referring to interpersonal processes (e.g., the value of pre-existing interpersonal relationships) or issues that are more of a practical/operational than social nature (e.g., the importance of receiving complete, accurate information). For example, was there any indication that positive interpersonal relationships paved the way for a sense of intergroup sharedness (as in the CSIMS model, an extension of ASPIRe), or merely that people work well with individuals they "like and trust"? Can we be certain that the Chairs established a sense of shared identity, or merely that they informed meeting participants of their respective roles and responsibilities (not quite the same thing)? 
The article could make a greater contribution by distinguishing between social identity and notnecessarily-social-identity phenomena, focusing on the latter (to the extent that adequate data exist), and pursuing clear social-identity-related themes in greater depth. For example, what does it mean if a crisisdriven heightening of fellow feeling (also observed in prior research) evaporates so quickly? Presumably the shared threat makes subgroup identities less salient and superordinate group identity more so, but what are the implications if this shift is so temporary/fragile, and is there any evidence that it can be made more enduring? The deeper the analysis can go, and the more an SIA analysis can be distinguished from a more generic interpretation, the greater the potential value. You might be interested in our article “Managing Intergroup Silos to Improve Patient Flow" (Health Care Management Review) - we too confronted a dataset in which many of the social processes discussed were not necessarily social identity processes, but chose to hone in on the latter.

Also, it would be helpful to have some more details about how the analysis was done - not merely what are the official steps in this version of thematic analysis, but who did what (did one author take the lead on coding and others review their work? did multiple authors code independently and then compare interpretations? etc.) and how the social identity approach was incorporated. Thematic analysis is typically an inductive approach, but the interpretations presented are theory-based. Did the coder(s) have some social identity themes in mind from the outset (more of a "directed content analysis" approach), did they incorporate social identity constructs as "sensitizing concepts," or indeed did they start the analysis without reference to the SIA and then decide it would be relevant? Further to methods, presentation of interview times down to the second seems too detailed, but it might be useful to briefly describe any implications/limitations associated with the disparate number of interviews per person. 